\title{
Photoinduced mass-transport based holographic recording of surface relief gratings in amorphous selenium films
}

\author{
M. L. Trunov, ${ }^{1, a)}$ P. M. Lytvyn, ${ }^{2}$ S. N. Yannopoulos, ${ }^{3}$ I. A. Szabo, ${ }^{4}$ and S. Kökényesi ${ }^{4}$ \\ ${ }^{1}$ Department of Engineering, Uzhgorod National University, Uzhgorod 88000, Ukraine \\ ${ }^{2}$ Institute of Semiconductor Physics, NAS of Ukraine, Kiev 03028, Ukraine \\ ${ }^{3}$ FORTH/ICE-HT, P.O. Box 1414, GR-26504 Patras, Greece \\ ${ }^{4}$ University of Debrecen, H-4010 Debrecen, Hungary
}

(Received 17 May 2011; accepted 27 June 2011; published online 2 August 2011)

\begin{abstract}
Surface relief gratings formation in amorphous selenium thin films in two recording configurations with light intensity modulation were studied in situ by real-time atomic force microscopy and diffraction efficiency measurements. We report observation of mass transport effect in films induced by band-gap irradiation when the light polarization of the recording beams has a component along the light intensity gradient (" $p-p$ " scheme of recording) that allows obtaining giant stable gratings in this versatile chalcogenide material. On the contrary, only a pure scalar weak grating caused by photoinduced volume shrinkage is obtained in the " $s-s$ " recording configuration, even for long-term irradiation. (C) 2011 American Institute of Physics. [doi:10.1063/1.3614432]
\end{abstract}

Chalcogenide glasses $(\mathrm{ChG})$ are known to exhibit a rich variety of photoinduced changes when illuminated with near bandgap light including changes in mechanical properties, termed as photoplastic effects. ${ }^{1}$ Among them, the effect of light induced surface deformation and direct fabrication of surface relief gratings (SRGs) in ChG is intensively studied over the last decade both experimentally ${ }^{2-10}$ and theoretically. ${ }^{11}$ Two main types of SRG induced by holographic recording, due to the excitation intensity modulation, with near band-gap light in $\mathrm{ChG}$ can be distinguished according to their formation mechanism and their properties: ${ }^{10}$ (1) small scalar SRG induced by either volume expansion or shrinkage due to different response of the material in the bright and dark zones of the interference pattern formed and (2) giant vectorial SRG induced by lateral mass transport in the case where the light polarization of the recording beams has a component along the light intensity gradient.

Experiments have shown that only few compositions of ChG demonstrate both types of SRG. For example, vectorial SRG have been observed only for Se-rich films in the binary As-Se system $\left(\mathrm{As}_{20} \mathrm{Se}_{80}\right)^{10}$ and for the compositions close to $\mathrm{As}_{40} \mathrm{~S}_{60}$ in As-S glasses ${ }^{3}$ while scalar SRGs are common to all glass compositions of As-S and As-Se glasses. While for As-S glass the above observation (lack of vectorial SRG) could be accounted for by considering the photo-induced polymerization effect in S-rich compositions, ${ }^{12}$ in the case of As-Se glasses the effect is not yet well understood. Although elemental amorphous $\mathrm{Se}$ (a-Se) is a model glass-former in chalcogenide science, no systematic studies of SRG formation have been carried out up to now, while investigations of other photoinduced effects abound. ${ }^{13} \mathrm{~A}$ fact that perplexes studies of SRG formation relates to the photoinduced crystallization of a-Se (Ref. 14) under band-gap illumination, thus preventing the formation of advanced surface relief stable in time.

Few papers report the formation of surface relief induced by holographic recording for a-Se films. ${ }^{2,5,15}$ In an early study, Haro-Poniatowski et al. ${ }^{15}$ employing phase conjugation, have

${ }^{\text {a)} E l e c t r o n i c ~ m a i l: ~ t r u n o v . m @ g m a i l . c o m . ~}$ shown that small $(20 \mathrm{~nm}$ in height) photoinduced surface relief gratings appears in $4 \mu \mathrm{m}$-thick films with the same spatial period. Slightly higher reliefs $(\sim 40 \mathrm{~nm})$ formed after prolonged exposure was reported in a later work. ${ }^{5}$ In these works, ${ }^{2,5,15}$ the main mechanism of SRGs generation is connected to the photoinduced volume changes of the film due to the localized structural rearrangements and ordering of a-Se chains, although no direct experimental evidence has been yet provided to support this conjecture. Some controversial results exist, however, concerning the question whether the formation of the SRG results in irreversible crystallization of a-Se after prolonged illumination ${ }^{5}$ or if the films region where the whole SRG forms is in the amorphous phase. ${ }^{2,15}$ Note also that a possible contribution of the polarization state of writing beams was not considered in previous studies.

In this paper, we present an in situ study of the SRG formation in a-Se thin films under holographic exposure by band-gap light with relative moderate intensity at the surface of the layer in different schemes of recording. We investigate how the polarization of the writing beams influences the type of SRGs forming under long-term exposure and demonstrate that $p-p$ polarized beams cause non-saturable mass transport whereas $s$-s polarized beams induces small, saturable scalar grating.

The experiments were performed on $2 \mu \mathrm{m}$ thick a-Se films deposited on glass substrates held at ambient temperature by thermal evaporation from bulk Se with a deposition rate of $3-5 \mathrm{~nm} \cdot \mathrm{s}^{-1}$. SRGs with a period of $\sim 3.6 \mu \mathrm{m}$ were holographically recorded at $300 \mathrm{~K}$ using as a writing source, a $20 \mathrm{~mW}$ linearly polarized laser operating at a wavelength of $650 \mathrm{~nm}(\mathrm{~h} v=1.9 \mathrm{eV})$, which is comparable with the band-gap energy of a-Se. The total light intensity on the surface of the samples was varied from $0.35 \mathrm{~W} / \mathrm{cm}^{2}$ to 20 $\mathrm{W} / \mathrm{cm}^{2}$. After splitting the main beam by a biprism beam splitter, two recording beams of equal power density interfere on the film free surface at the incidence angle $\theta\left(\theta \sim 5^{\circ}\right)$ as is shown in Fig. 1. The incidence angle of $5^{\circ}$ yields a spatial interference period of $\sim 3.6 \mu \mathrm{m}$ along the $x$-axis. Note that for such angle, the difference in reflectivity for $s$ - and 
$p$ - polarized beams is negligible thus the same amount of energy transferred to the film for both polarizations.

The time evolution of the surface topography of the film under illumination was investigated by a NanoScope III atomic force microscopy (AFM) (Veeco, Inc.). We have exploited, depending on the traces of AFM tip, real 3D surface topography images and virtual 3D surface profile (the shape transformation of one scan line). The slow scan direction was disabled in the last case and thus the ordinate in the images shown in the figures reflects the time scale (see arrows titled as "Time"). The evolution of diffraction efficiency (the ratio of intensities of the first diffracted beam to the incident one) was measured using a violet laser beam $(\lambda=406 \mathrm{~nm}, I=10$ $\mu \mathrm{W})$ and was taken proportional to the intensity variation of the first diffraction peak in reflection mode.

Two types of experiments on SRG recording were carried out using (1) $p$ - $p$ polarized recording beams and (2) $s-s$ polarized ones. Fig. 1 illustrates the results for the SRGs kinetics formation at light intensity of $20 \mathrm{~W} / \mathrm{cm}^{2}$. The initial surface is flat as it is shown at the bottom of the images, Figs. 1(a) and 1(b). A sudden turning on of the writing beams results in photocontraction of the film region illuminated by the bright fringes of interference pattern. This gives rise to the instantaneous SRGs formation with approximately equal negative amplitude that reach simultaneously a maximum value of $-15 \mathrm{~nm}$ after a short time $(20 \mathrm{~s})$ of exposure irrespectively from the scheme of recording [Fig. 1(c)]. Note again that these SRGs are out of phase regarding to the light intensity profile which is opposite to the previous observa-

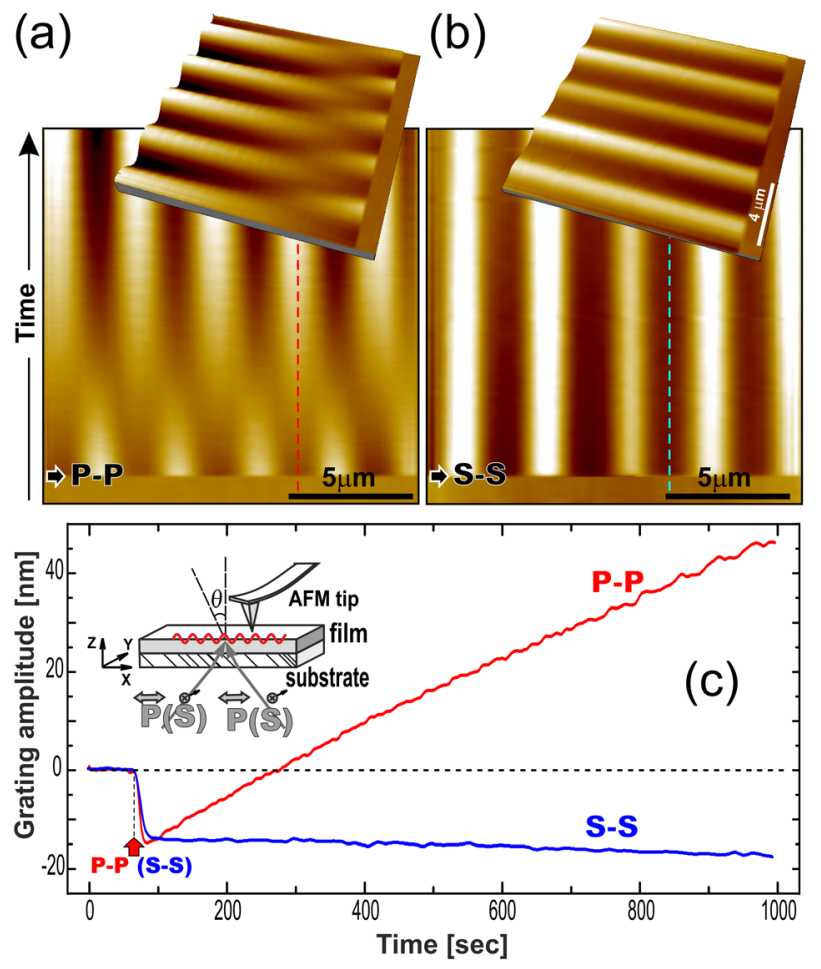

FIG. 1. (Color online) The dynamics of SRG growth in different polarization schemes of inscription: 2D images of SRG evolution with appropriate 3D reconstruction (a) and (b); SRGs amplitudes as a function of time (c). SRGs amplitudes were measured along the vertical solid lines in (a) and (b). The arrows indicate the moments when recording beams are switched on. Polarizations of the beams are indicated directly in the images (a)-(c). The scheme of experiment is illustrated by the inset in (c). tion in $\mathrm{As}_{20} \mathrm{Se}_{80}$ composition. ${ }^{10}$ However, the negative value of the amplitude of the SRG obtained in $p$ - $p$ scheme of recording decreases sharply and then vanishes very fast [see Fig. 1(c), negative part of p-p curve]. With further light exposure, the onset of the main grating takes place but this grating appears with a half-period phase shift and produces a relief in direct phase in relation to the light pattern. This half-period phase shift with respect to the interference pattern at the transition between those two SRGs is clearly seen from the corresponding image [Fig. 1(a)]. Accordingly, the kinetics of the SRG formation in $p-p$ polarized beams experiences a sign change and increases linearly in the course of exposure without explicit saturation [Fig. 1(c), positive part of $p$ - $p$ curve]. On the contrary, at the $s$-s scheme of recording only one SRG is formed, out of phase regarding the interference pattern, Fig. 1(b), with rather small but stable amplitude under prolonged light exposure [Fig. 1(c), s-s curve].

These results clearly demonstrate that two distinct mechanisms are responsible for SRGs formation in a-Se at different schemes of recording: (1) photoinduced volume shrinkage (it generates fast, but small SRG, type I, with negative amplitude values) and (2) photoinduced lateral mass-transport which takes place only for $p$ - $p$ polarized writing beams (it generates relatively slow, but non-saturable SRG, type II, with positive amplitude values). It would be instructive to comment on the fact that these results were not expected in view of the following reasons: (1) the photoplastic effect ${ }^{1}$ which is one of the important conditions of mass-transport appearence, ${ }^{10}$ should, in principle, be very weak in a-Se due to low glass formation temperature $\left(\mathrm{T}_{\mathrm{g}} \approx 40^{\circ} \mathrm{C}\right)$ and (2) photocrystallization may also occur for long-term irradiation. ${ }^{16,17}$ The last process would impede the displacement of material over atomic distances and hence the SRG height should reach saturation levels over an extended time of laser irradiation, which contradicts the linear height vs. time dependence observed experimentally [Fig. 1(c), positive part of p-p curve].

For different light power densities ranging from $0.35 \mathrm{~W} /$ $\mathrm{cm}^{2}$ to $20 \mathrm{~W} / \mathrm{cm}^{2}$, we have observed the same behavior as presented in Fig. 1, moreover at low intensity $\left(0.35 \mathrm{~W} / \mathrm{cm}^{2}\right)$ additional details of the process were discovered. Fig. 2(a) shows a series of cross-sections kinetics of transition of SRG I into SRG II at the $p-p$ scheme of recording. These crosssections were extracted from the set of five sequential AFM scans merged into the common time scale in Fig. 2(b) (for a total exposure of $107 \mathrm{~min}$ ). Two main observations emerge: (1) the process involves an intermediate regime where valleys transform to M-shaped peaks and (2) the development of severe roughness on the peaks of SRGs with a "droplet" size of $\sim 150 \mathrm{~nm}$ in diameter and of 3-5 nm in height, see Fig. 2(c). None of these observations has been reported up to now for thermally deposited a-Se films. It is worth-noting that the roughness pattern tends to form perpendicularly to the electric vector of $p$-polarized writing beams [Figs. 2(b) and 2(c), insets]. Preliminary micro-Raman studies along the grating vector of SRG, however, exclude the presence of crystallites; more detailed Raman scattering investigations are currently underway. The origin of the transiently induced roughness is still speculative. Roughness has not been reported in single-beam band-gap irradiation even at long times. It remains to be confirmed if this effect appears only 


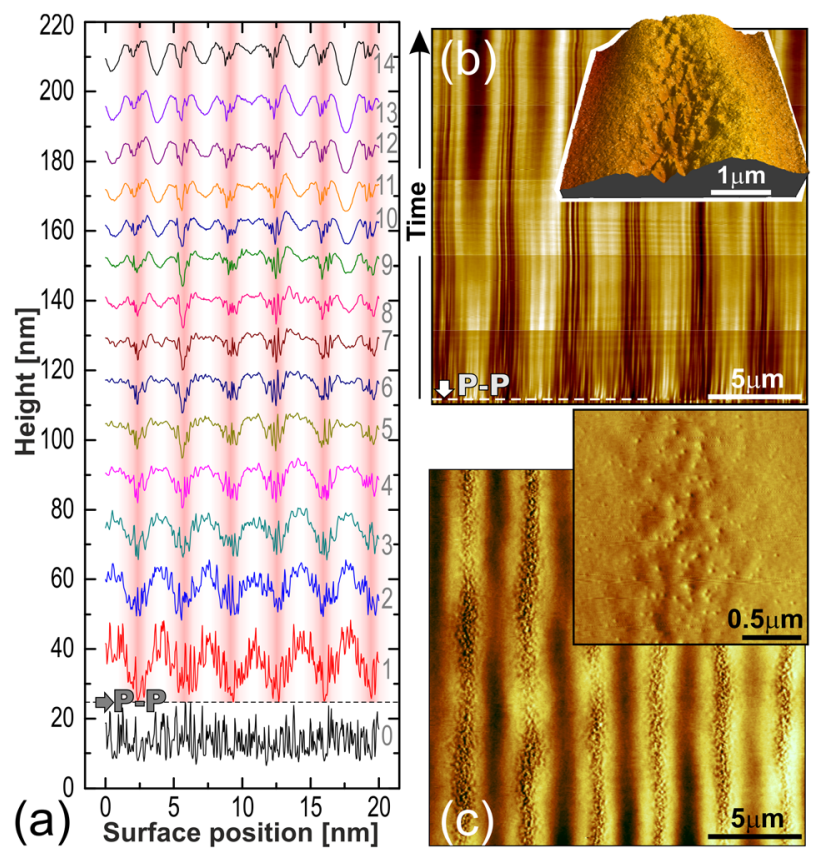

FIG. 2. (Color online) The dynamics of SRG growth induced by $p-p$ polarized writing beams: the shapes of SRGs (a) extracted from the 2D image of SRG evolution (b) at different exposure times $(0-$ before exposure, $1-$ at $38 \mathrm{~s}, 2$ - at $690 \mathrm{~s}, 3-14$ - with the interval of $470 \mathrm{~s}$ up to $107 \mathrm{~min}$ of exposure); 3D AFM image of the SRG after exposure of $130 \mathrm{~min}$ (c). Enlarged images of the "droplets" at the peak of SRG are shown in the insets (b) and (c). The vertical pattern corresponds to the bright fringes in the light intensity profile in (a).

in the holographic recording scheme as a mass rearrangement over local spatial scale, which is the intermediate step for SRG II formation. Detailed Raman investigations might provide evidence about the role of holographic recording on the chains-rings equilibrium in a-Se and the interactions of the polymeric chains.

Several studies have shown that photocrystallization occurs in a-Se films under long-term exposure by singlebeam bandgap light. ${ }^{16,17}$ On the contrary, in the present investigation of holographic recording, we observe decrease of the roughness instead of its evolution to crystallites. SRG I transforms into transient light-induced SRG with M-shape profile, which is further replaced by SRG II (see Fig. 3). The latter has a sinusoidal surface profile during the whole course of exposure where mass transport takes places. The various insets in Fig. 3 present the corresponding cross-sections for these SRGs (as pointed out by arrows A and B in Fig. 3) and the initial part of diffraction curve in an enlarged time scale. The amplitude of the light-induced SRG II substantially increases in accordance with increasing of the first-order diffraction: typically the grating height increases up to $\sim 200$ $\mathrm{nm}$ after $10 \mathrm{~h}$ of exposure which corresponds to diffraction maxima (Fig 3, solid curve). Further exposure generate further growth of SRG in height that is limited by film thickness only (not shown here).

Pheomenological explanations of the light-induced mass transport effect in ChGs were given recently ${ }^{3,18}$ based on the continuous creation of dipolar defects or other anisotropic structural units. In a-Se anisotropic units can be envisaged as branched Se chains through over-coordinated Se atoms. A recent experimental and theoretical study revealed that the

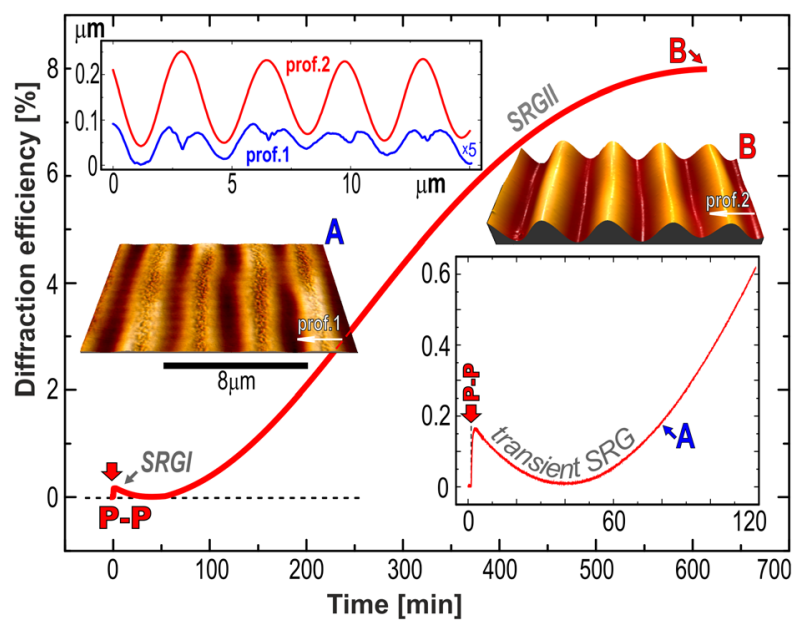

FIG. 3. (Color online) Diffraction efficiency during the SRG formation by $p$ - $p$ writing; in the corresponding insets: the shapes of SRGs at different time of exposure (marked as profiles in appropriate 3D AFM images) which correspond to different stages of SRG formation. Note that the height scale in Prof. 1 is enlarged five times compared to Prof. 2.

surface of a-Se is dominated by hypervalent defects, i.e., three-fold and four-fold coordinated Se atoms. ${ }^{19}$ The scission of the weak bonds of such over-coordinated atoms offer the possibility of photoinduced mass-transport and SRG formation in a-Se.

In summary, we report on two distinct mechanisms of SRG holographically recorded in a-Se depending on the polarization of writing beams. In the $s$-s scheme of recording, scalar SRGs appears through the photoinduced volume contraction. For $p-p$ polarized beams, lateral mass-transport takes places altering the mechanism of SRG formation and, in turn, generates giant non-saturated SRGs. Consequently, polarized light induced effects of SRG formation by holographic recording in amorphous chalcogenides ${ }^{3,6,8-10,18}$ can be realized in the simplest material among ChG. This may lead to a better understanding of photoinduced mass-transport on $\mathrm{ChG}$ thus enabling the fabrication of photonic elements.

This work is supported by the TAMOP 4.2.1./B-09/1/ KONV-2010-007 project, which is co-financed by the European Union and European Social Fund.

${ }^{1}$ S. N. Yannopoulos and M. L. Trunov, Phys. Status Solidi B 246, 1773 (2009).

${ }^{2}$ J. M. Bellisteros et al., Appl. Phys. A 65, 463 (1997).

${ }^{3}$ A. Saliminia et al., Phys. Rev. Lett. 85, 4112 (2000).

${ }^{4}$ M. Fischer et al., Synth. Met. 127, 303 (2002).

${ }^{5}$ V. Palyok et al., Appl. Phys. A 74, 683 (2002).

${ }^{6}$ K. E. Asatryan et al., Appl. Phys. Lett. 84, 1626 (2004).

${ }^{7}$ Zh. Yang et al., J. Phys. D: Appl. Phys. 42, 135412 (2009).

${ }^{8}$ M. Trunov et al., Proc. SPIE 7598, 75981H-1 (2010).

${ }^{9}$ U. Gertners and J. Teteris, Opt. Mater. 32, 807 (2010).

${ }^{10}$ M. L. Trunov et al., Appl. Phys. Lett. 96, 111908 (2010).

${ }^{11}$ Yu. Kaganovskii et al., Appl. Phys. Lett. 97, 061906 (2010).

${ }^{12}$ S. N. Yannopoulos et al., Opt. Lett. 36, 534-536 (2011).

${ }^{13}$ S. Kasap et al., Phys. Status Solidi B 246, 1773 (2009).

${ }^{14}$ J. Dresner and G. B. Stringfellow, J. Phys. Chem. Solids 29, 303 (1968).

${ }^{15}$ E. Haro-Poniatowski et al., Opt. Lett. 17, 252 (1992).

${ }^{16}$ S. G. Hansen and T. E. Robitaile, Thin Solid Films 151, 111 (1987).

${ }^{17}$ V. K. Tikhomirov et al., J. Non-Cryst. Solids 227-230, 732 (1998).

${ }^{18}$ M. L. Trunov et al., Appl. Phys. Lett. 97, 031905 (2010).

${ }^{19}$ T. Scopigno et al., Nat. Commun. 2, 195 (2011). 